August $2005 \cdot \underline{\text { Volume } 99} \cdot \underline{\text { Number } 8}$

\title{
Self-evaluation and Recruitment of Feedback for Enhanced Social I nteraction by a Student with Visual I mpairment
}

Divya Jindal-Snape

Abstract: A student who is visually impaired was trained to evaluate his social behavior and to recruit feedback from his sighted peers, who were trained by him to provide the feedback. The self-recruitment of feedback improved the student's accuracy in evaluating social skills requiring visual cues. In addition, the peers extended their feedback to other aspects of the social environment than social behavior.

Visual impairment (that is, blindness or low vision) can affect a child's social development and the formation of friendships with sighted peers (Rosenblum, 1998). One factor that inhibits a child's social development and formation of friendships is the lack of visual cues. According to MacCuspie (1996, p. 37), "Limited access to both learning and using visual cues can be observed to detract from the social development of children with visual impairment from a very early age." Similarly, nonverbal communication and feedback, which are important components of social interaction, are available to sighted children but are not easy for children who are visually impaired to follow. It has been observed that unless they are prompted, significant others in the environment (such as teachers, peers, and family members) fail to supply feedback that is meaningful to individuals who are visually impaired (Jindal-Snape, 2004). The lack of such feedback to reinforce appropriate behaviors or to discourage those that are 
seen to be inappropriate puts visually impaired children at a disadvantage (MacCuspie, 1996) in attaining social skills, such as initiating and maintaining interactions and using the direction of gaze to show a willingness to engage in social interactions (McGaha \& Farran, 2001).

Self-evaluation has been observed to be effective in increasing the social interaction and generalization and maintenance of the social behavior of children with disabilities (Webber, Scheuermann, McCall, \& Coleman, 1993), including those with visual impairments, and their peers (Jindal-Snape, 2004, 2005; Jindal-Snape, Kato, \& Maekawa, 1998). However, for skills that require visual cues, feedback from the significant others in the visually impaired child's environment is crucial (Jindal-Snape, 2004, 2005). Such feedback also has an effect on the ability of children with visual impairments to evaluate their own social behavior that requires visual cues (Jindal-Snape, 2004). Schloss and Smith (1994) suggested that many behaviors are difficult for individuals to self-monitor and to detect changes in. In such cases, feedback to guide self-evaluation has to be provided by significant others in the environment.

With these points in mind, as previously reported (Jindal-Snape, 2005), I trained a sighted peer to give feedback about the social behavior of a visually impaired boy through reinforcement. This feedback from the peer led to the enhancement of the boy's social interaction and accuracy of self-evaluation. Furthermore, not only did the sighted peer extend feedback to other peers who were visually impaired, but other sighted peers began to provide feedback to the boy who was visually impaired without being trained to do so.

However, I concluded that for the feedback to be maintained and to be provided in a meaningful way, it might be better for somebody other than a researcher to train the sighted peers to 
provide it, because somebody in the natural environment would be in a better position to maintain the behavior (Jindal-Snape, 2005). The best person to do so would probably be the child who required this feedback. Mank and Horner (1987) suggested that the self-recruitment of feedback from the external environment may contribute to its maintenance. Hence, in the study reported in the current article, a boy who was visually impaired and who was self-evaluating his social skills was trained to recruit feedback.

The main purpose of this study was to train peers in the boy's environment to give feedback and to determine the effect of this feedback on the boy's social skills and accuracy of his selfevaluation. Other purposes were to observe whether the recruitment of feedback generalized across settings and peers and to determine whether, after learning to provide feedback to facilitate self-evaluation, the peers would also learn to give unprompted information about the social environment.

Informed consent was obtained from all the children who participated in the study, as well as from their parents, the classroom teacher, and the head teacher. The names of the children were changed to protect their confidentiality.

\section{Method}

\section{Setting}

The study was conducted in a class of 30 children in an integrated school in New Delhi, India, that included 6 visually impaired children. Although the teacher had no formal qualifications for teaching children who are visually impaired, she had the support of the school's resource person, who is qualified in the field of visual impairment. The seats were arranged in rows, with 3 children at each table. Each child with a visual impairment sat with 2 sighted children. According to the class teacher, this 
arrangement was made so that the sighted children could read out things from the blackboard or books to the children who were visually impaired.

\section{Participants}

Sameer (a boy who was totally blind) and four sighted peerstwo boys, Mohan and Rakesh, and two girls, Meena and Sunitaparticipated in the study. However, the data of two of the peers, Mohan and Meena, are only given here because they were present in the training sessions during which Sameer was encouraged to request feedback from his peers on direction of gaze. Rakesh and Sunita were present during the sessions in which Sameer evaluated himself in relation to conversation. Sameer, Mohan, and Meena were aged 9 years, 4 months; 9 years; and 9 years, 2 months, respectively.

\section{Sameer}

The social age of Sameer was 5 on the Vineland Social Maturity Scale (Indian adaptation by Malin and Raj, 1992). The teacher reported that Sameer did not participate in class activities and did not interact with his sighted peers; he did not initiate conversations and either ignored initiations by others or laughed without responding. When the teacher addressed Sameer, he just responded briefly. The teacher said that Sameer usually gave no indication of even listening to other people's conversations and was becoming increasingly isolated. However, she noted that he interacted with one peer who was also visually impaired.

Sameer lived in a hostel (known as a "residential preparatory school") and went home about once a month. The director of Sameer's hostel reported that Sameer had joined the preparatory school five years before and had been attending the integrated education school for the past three years. Sameer did not 
participate in the activities at the hostel, did not respond to initiations by the other children, and played with just one visually impaired friend (the same one mentioned by the teacher). When that child went home on the weekend, Sameer kept to himself.

Sameer's failure to respond to his peers was having an adverse effect on his relationship with his peers, who, even when prompted, soon stopped interacting with Sameer. Therefore, it was considered important for Sameer to show that he was paying attention to his peers - that he not only oriented his gaze in their direction, but responded to their initiations. Furthermore, it was important for him to learn to approach the peers, draw their attention, and continue to converse with them.

\section{Sighted peers}

Both Mohan and Meena lived with their families. Mohan was a cheerful boy who willingly helped other children and was attentive in class. Meena was the monitor of the class and participated actively in various activities. Mohan and Meena were selected because they were recommended by their teacher as being socially active.

Mohan and Meena were observed in the classroom and playground to gesture extensively while speaking, use their hands to show the size of objects during conversations, stop speaking if other children joined their group without telling Sameer, explain riddles or locations by drawing shapes or routes with their fingers on their own hands, and to nod their heads instead of answering yes or no. Zanandrea (1998) noted that such unpredictable movements of sighted peers without any accompanying verbal communication may confuse children with visual impairments.

\section{Target Behaviors}


On the basis of interviews and observation, I chose two target behaviors for Sameer: modification of the direction of gaze (that is, orientation of the face or body toward others) and an increase in conversation (see Box 1). The target behaviors for Mohan and Meena were to give feedback regarding Sameer's direction of gaze and any other feedback that Sameer might prompt them to give (see Box 2). The nontarget behavior for Mohan and Meena was to give information related to the social environment through auditory or physical cues; for example, telling Sameer if somebody entered the room.

\section{Study design and procedures}

The study covered a period of nearly 6 weeks (including prior observation, interviews with the teacher and director, and 10 days' absence on Sameer's part). Two 10-minute sessions were conducted daily from Monday to Friday, one for direction of gaze and one for conversation. The settings for both sessions were different, and there was a gap of a couple of hours between them. There was a gap of 10 days in training after the 11th session for both behaviors when Sameer was absent from school for family reasons. A multiple baseline design across behaviors was used for Sameer.

\section{Direction of gaze}

There were three phases for direction of gaze-self-evaluation only, self-evaluation and recruitment of feedback, and selfevaluation only. In the first and third phases (both self-evaluation only), no feedback was to be solicited by Sameer. In the second phase (self-evaluation and recruitment of feedback), Sameer was asked to prompt Mohan or Meena to provide feedback about the accuracy of his direction of gaze.

Sameer, Mohan, and Meena were asked to sit and chat or play 
freely. Each session lasted 10 minutes. After the target behavior was described to Sameer, at every 3-minute interval, Sameer was cued to evaluate verbally whether he thought he was facing the person with whom he was speaking. I cued him to self-evaluate by tapping my foot twice, a cue that Sameer agreed to before the intervention started. I tapped my foot so that I did not stop the conversation or play, and Sameer was able to make his selfevaluations after the completion of that particular conversation or activity. Mohan and Meena received no formal training for providing feedback. They were instructed by the researcher to provide Sameer with feedback whenever he recruited feedback from them.

The remainder of the session proceeded without any comment from me. The first phase (self-evaluation) lasted for 4 sessions, the second phase (self-evaluation and self-recruitment of feedback) lasted for 6 sessions, and the third phase (selfevaluation) lasted for 3 sessions. Afterward, 5 sessions of a generalization probe were conducted for direction of gaze.

As a result of the three phases for Sameer, there were three phases for Mohan and Meena: no prompt for feedback, prompt for feedback, and no prompt for feedback. These phases were followed by a generalization probe for Mohan and Meena to observe whether they would give unprompted feedback to another child who was visually impaired. As was mentioned earlier, giving information about the social environment was Mohan and Meena's nontarget behavior.

\section{Conversation}

The training for conversation was conducted in a different setting from the training for direction of gaze, and with two different sighted peers: Rakesh and Sunita. Eleven sessions of selfevaluation were conducted for conversation. Sameer was cued for 
self-evaluation in the same manner as for direction of gaze. Then five sessions of a generalization probe were held for conversation.

\section{Interobserver reliability}

All the sessions were videotaped, so that the behaviors could be independently coded by the observers. I and two special education undergraduate students who were in their final year later observed the videotapes. All the observers used the same definition of the target behaviors. Five minutes of the 10-minute sessions were observed, from the 3rd to the 8th minute of each session. (Accuracy of self-evaluation was observed during all 10 minutes of the session.) Interobserver reliability was calculated using the formula, number of agreements divided by number of agreements plus number of disagreements, multiplied by 100. Interobserver reliability for direction of gaze for Sameer was an average of $86 \%$ during the baseline; $86 \%, 84 \%$, and $90 \%$ during the three phases, respectively; and $88 \%$ during the generalization probe. For conversation, interobserver reliability was $99 \%$ during the baseline, $100 \%$ during the self-evaluation phases, and $100 \%$ during the generalization probe.

\section{Results}

\section{Sameer}

\section{Direction of gaze}

As can be seen in Figure 1, at the baseline, direction of gaze was 0 , that is, Sameer was never observed to look or turn toward his peers. During the first phase (self-evaluation only), it reached an average of 129 seconds out of a possible total of 300 seconds. Direction of gaze reached an average of 208 seconds during the second phase (self-evaluation and self-recruitment of feedback) and was maintained at an average of 205 seconds during the third 
phase (self-evaluation with no prompt for recruitment of feedback), despite the fact that there was a gap of 10 days between the two phases. In the third phase, Sameer was observed to recruit feedback as well (without any prompt from me). During the generalization probe, direction of gaze was at an average of 194 seconds.

\section{Conversation}

At the baseline, Sameer conversed for an average of 108 seconds. However, most of the time he was a passive listener. By the second session of the self-evaluation phase, his conversation had reached the maximum possible of 300 seconds, with active participation; that is, Sameer was observed to ask questions, respond to peers' questions, lead the conversation, and help maintain a smooth flow of the conversation. Apart from the 249 seconds of the first session, his conversation remained at more than 290 seconds at all times and was mostly at 300 seconds. The average of all the 11 sessions was 291 seconds, and for the last 10 sessions, it was 296 seconds. During the generalization probe, Sameer's conversation was maintained for an average of 297 seconds. Although Sameer was never asked to recruit feedback during the training for conversation (which was carried out with different peers in a different room), he recruited feedback. However, this feedback was related to the direction of his gaze, not his conversation. Social interaction between Sameer and his peers increased considerably.

\section{Direction of gaze in the conversation setting}

As noted, Sameer started to recruit feedback about his direction of gaze in the conversation setting from his peers. Data for Sameer's direction of gaze was recorded for 8 of these sessions to document the generalization of skills across subjects and to see whether Sameer would continue to recruit feedback from them as 
well. Direction of gaze was found to be appropriate for an average of 201 out of 300 seconds. Sameer was observed to recruit feedback from these peers continuously. This finding shows that not only the skill, but the recruitment of feedback, had generalized.

\section{Recruiting feedback}

Sameer recruited feedback about the appropriateness of his direction of gaze during the second phase of the study, when he was also self-evaluating his direction of gaze, as prompted by me to self-evaluate. However, he was observed to ask peers for feedback even after this phase was over. Again, during the same period of time as the second phase of direction of gaze, he started recruiting feedback about his direction of gaze during the conversation sessions and was observed to continue to do so throughout the rest of the study. He never asked for feedback for skills that were related to conversation. Furthermore, as mentioned earlier, recruitment of feedback generalized to other peers as well.

\section{Accuracy of self-evaluation}

To assess the accuracy of self-evaluation, the entire session was observed. During the self-evaluation phase of direction of gaze, Sameer evaluated himself accurately an average of $41 \%$ per session. During the self-evaluation and self-recruitment of feedback phase, the accuracy of self-evaluation of direction of gaze increased to an average of $78 \%$ per session. It is to be noted that the accuracy was lower in the first two sessions. In the later sessions, Sameer evaluated himself accurately $100 \%$ of the time.

In the third phase - that is, with no prompting to recruit feedback - Sameer continued to ask for feedback and self-evaluated accurately an average of $100 \%$ per session. It is to be remembered 
that there was a gap of 10 days between the second and the third phase, and that no training took place in that period.

\section{Mohan}

In the self-evaluation and recruitment of feedback phase for direction of gaze, Sameer recruited feedback three times in a session, since he was cued after every three minutes. It was observed that he usually asked Mohan, rather than Meena, for feedback regarding his direction of gaze. Mohan provided feedback on direction of gaze only when prompted. However, he started providing unprompted information about the social environment in ways that were understandable to Sameer. The provision of unprompted information was calculated as a percentage (the number of times such information was given, divided by the total opportunities to give that information in that session, multiplied by 100). From the initial level of 0 , the provision of unprompted information reached an average of more than 90\% (see Figure 2). At times, he was also observed to prompt Meena to explain riddles and other such things on Sameer's hand.

After the completion of the training with Sameer, Mohan was observed with another child with a visual impairment for two sessions. During this generalization probe, Mohan gave feedback at a rate of two times per session. Furthermore, Mohan provided information about the social environment in an appropriate way throughout.

\section{Meena}

Sameer rarely asked Meena for feedback, except once when Mohan was not there, but Meena was observed to provide unprompted feedback and, at times, added to the feedback provided by Mohan. However, Meena continued to gesture and 
explain something to Mohan through visual cues, with no physical or auditory explanation for Sameer. Only after she was prompted twice by Mohan did she start giving information about the social environment in an appropriate way. Afterward, she was observed to provide information in this way an average of $50 \%$ of the time.

\section{Discussion}

Mank and Horner (1987) found that a self-recruited feedback strategy that included self-monitoring and recruiting of feedback was effective not only in increasing social skills, but in maintaining them. In their study, after self-monitoring, the participants went to their supervisor with their self-monitoring data to ask for feedback. This finding implies that the participants recruited feedback after deciding whether the social behavior had occurred or not. However, feedback that is provided in this delayed manner is not natural and may not even be necessary when the consequences are clear. That is, in the case of the participants in Mank and Horner's study, meeting the criteria of producing the target behavior should have served as feedback. The findings of the current study partially support Mank and Horner's findings, in that they revealed that although selfevaluation alone could increase the duration of a desirable direction of gaze, the duration was further enhanced by the selfrecruitment of feedback. However, feedback was recruited before the self-evaluation was verbalized. Sameer recruited feedback not only when prompted, but in the next phase when no prompt was given. In addition, the generalization of the recruitment of feedback was easy, as can be seen by the fact that Sameer recruited feedback from other peers. Self-recruited feedback also helped Sameer to evaluate his behavior accurately.

It was interesting to observe that although the self-recruitment of feedback generalized to other peers and settings, Sameer never 
recruited feedback for skills related to conversation. This finding suggests that for the verbal skills like the ones needed for conversation, the consequences are clearly understandable and themselves serve as feedback, so that no external feedback is necessary.

Sameer's conversation increased immediately after the introduction of self-evaluation. His self-evaluation was also accurate from the beginning. Sameer was never prompted to recruit feedback in the sessions in which he was self-evaluating in relation to conversational skills. Furthermore, these sessions were carried out with peers other than the ones who participated in the training for direction of gaze. Nevertheless, Sameer was observed to recruit feedback for direction of gaze during these sessions. He did not recruit feedback for other skills related to conversation even once. This finding suggests that Sameer did not require feedback from peers for conversation but required it for direction of gaze. It is also possible that Sameer realized the importance of appropriate direction of gaze in conversation.

As was seen in previous studies (Jindal-Snape, 2004; JindalSnape et al., 1998) self-evaluation was effective in generalizing social skills and increasing social interaction. In this study it was also shown to be effective in generalizing skills and interaction across participants and settings. During the generalization probe, the social skills stayed at nearly the same levels as during the intervention, and use of appropriate direction of gaze generalized to situations with other peers as well. In addition, Sameer was also observed to recruit feedback from these peers.

Workman (1986) suggested that children who are visually impaired face obstacles in obtaining the full benefit of interaction with peers because of their visual limitations. These children miss many important nonverbal cues given by their teachers and peers through facial expressions, gestures, and body language. 
Furthermore, since they must rely almost exclusively on verbal cues, it is important to determine what type of cues can be provided by the teacher, peers, or others in the environment. Explaining visual information through auditory or physical means (such as explaining riddles through gestures) was observed as nontarget behavior. There was a distinct change in the behavior of the peers as well. Although Mohan never gave feedback related to direction of gaze unless prompted by Sameer, he started to give general information about the social environment without any prompting. Although he was seen to use terms like "there" and "this big," at the beginning of the study, he did not use them after the recruitment of feedback phase. Furthermore, he started to explain things by holding Sameer's hands and expanding them to show the size of an object, explaining the shape of a thing by drawing it on Sameer's hand with his finger, and so forth. This finding suggests that being prompted to provide feedback for direction of gaze may have served as a cue for Mohan to provide information that is attainable through vision in ways that were more desirable to Sameer.

Meena was observed to model Mohan. She started to provide feedback even though she was never prompted by Sameer. She also started to provide information about the social environment, although to a lesser extent than did Mohan. However, this finding suggests that modeling the provision of feedback and information about the social environment is feasible and that teachers need to provide more feedback and information to children who are visually impaired, so that other children may model the teacher and will start providing feedback and information without any formal training.

The findings of this study clearly show the need to provide feedback to students who are visually impaired in social situations to facilitate self-evaluation and the modification of social skills that require visual cues. Furthermore, they suggest that for skills 
that do not require visual cues, such as those that are related to conversation, self-evaluation can be effective on its own and is easy to do on the basis of consequences of such behavior. The results also suggest that self-recruitment of feedback from others is an effective way of gaining this feedback, since it generalizes to other settings and other peers.

Asking Sameer to evaluate himself every three minutes was essential because a longer time frame might have affected the accuracy of his self-evaluation. However, this time span may sometimes hinder the flow of conversation. Thus, it is worth trying to increase the duration in later sessions. Similarly, although the baseline for Sameer's direction of gaze ideally should have been longer, intervention was started after one session on the basis of observation in other settings and interviews before the study began. However, it is recommended that data for the baseline phase should be recorded for three to four sessions before an intervention begins.

\section{Conclusion}

Verbal feedback was effective in enabling the accurate selfevaluation of social skills that require visual cues. It was found that the accuracy of self-evaluation and of the target behavior improved considerably after the peers provided feedback to Sameer. However, once Sameer learned to perform the target behavior and to evaluate himself accurately, he could continue to do so even in the absence of feedback. This finding suggests that after the significant others in the environment provide the feedback initially, children can generalize and maintain their behavior even in the absence of these significant others.

Hence, it can be concluded that feedback from significant people in the environment is a way to help students who are visually impaired to develop social skills that require visual cues and that 
these people should make an effort to provide it accurately and in an appropriate way. However, it is important to discuss the most effective way of extracting this feedback from the environment. In this study, it was found that peers were better at giving feedback when prompted by Sameer, rather than when they were reinforced by me, as in a previous study (Jindal-Snape, 2005). Furthermore, self-evaluation procedures may also be applied to train the significant others in the environment to provide feedback.

Compared to the child in the previous study (Jindal-Snape, 2005), Sameer evaluated himself more accurately and modified his behavior much more than the other child. It is possible that while recruiting feedback, Sameer felt more in control than did the other child, to whom feedback might have seemed to be imposed. Another interesting observation was that in both cases peers who had not been trained also learned to give feedback and that they may have done so through modeling. This finding suggests that if such feedback and information were provided by the teacher in day-to-day life, the students might also model it.

Although neither group of peers provided any information about the social environment at the beginning of the study, Sameer's peers started to give such information to a greater extent by the end of the study than did those in the previous study (JindalSnape, 2005). It is possible that they did so because the situation was more natural, since I did not prompt the peers, as I did in the previous study. It may be interesting to investigate this possibility, especially because information about the social environment is important for the development of social skills.

It is also important to emphasize that sighted peers need to understand why children with visual impairments do not use visual cues. They need to be aware that visually impaired children pay attention to language, tone of voice, the proximity of the speaker, and the like. Despite the importance of inclusion and 
social interaction, there has been little research on the role of the environment and the development of social skills of significant others in the environment in relation to children with visual impairments. The findings of this study reinforce the notion that for effective interaction and inclusion, it is necessary to increase the repertoire of social skills of both children with visual impairments and their sighted peers.

\section{References}

Jindal-Snape, D. (2004). Generalization and maintenance of social skills of children with visual impairment: Self-evaluation and role of feedback. Journal of Visual Impairment \& Blindness, 98, 470-483.

Jindal-Snape, D. (2005). Use of feedback from sighted peers in promoting social interaction skills. Journal of Visual Impairment \& Blindness, 99, 403-412.

Jindal-Snape, D., Kato, M., \& Maekawa, H. (1998). Using selfevaluation procedures to maintain social skills in a child who is blind. Journal of Visual Impairment \& Blindness, 92, 362-366.

MacCuspie, P. A. (1996). Promoting acceptance of children with disabilities: From tolerance to inclusion. Halifax, Nova Scotia: Atlantic Provinces Special Education Authority.

Malin, A. J., \& Raj, J. B. (1992). Vineland Social Maturity Scale and manual: Indian adaptation. Mysore, India: Swayamsiddha Prakashana.

Mank, D. M., \& Horner, R. H. (1987). Self-recruited feedback: A cost-effective procedure for maintaining behavior. Research in Developmental Disabilities, 8, 91-112.

McGaha, C. G., \& Farran, D. C. (2001). Interactions in an 
inclusive classroom: The effects of visual status and setting. Journal of Visual Impairment \& Blindness, 95, 80-94.

Rosenblum, L. P. (1998). Best friendships of adolescents with visual impairments: A descriptive study. Journal of Visual Impairment \& Blindness, 92, 593-608.

Schloss, P. J., \& Smith, M. A. (1994). Increasing appropriate behavior through related personal characteristics. Applied behavior analysis in the classroom. Boston: Allyn \& Bacon.

Webber, J., Scheuermann, B., McCall, C., \& Coleman, M. (1993). Research on self-monitoring as a behavior management technique in special education classrooms: A descriptive review. Remedial and Special Education, 14, 38-56.

Workman, S. H. (1986). Teachers' verbalizations and the social interaction of blind preschoolers. Journal of Visual Impairment \& Blindness, 80, 532-534.

Zanandrea, M. (1998). Play, social interaction, and motor development: Practical activities for preschoolers with visual impairments. Journal of Visual Impairment \& Blindness, 92, 176-188.

Divya Jindal-Snape, Ph.D., lecturer, Faculty of Education and Social Work, University of Dundee, Gardyne Road Campus, Broughty Ferry, Dundee DD5 1NY, Scotland; e-mail: < $\underline{\text {. }}$ jindalsnape@dundee.ac.uk>.

\section{$\underline{\text { Previous Article | Next Article | Table of Contents }}$}

JVIB, Copyright (C) 2005 American Foundation for the Blind. All rights reserved. 


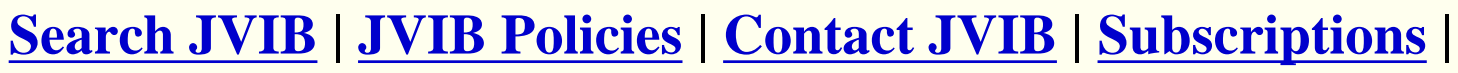 JVIB Home}

If you would like to give us feedback, please contact us at jvib@afb.net.

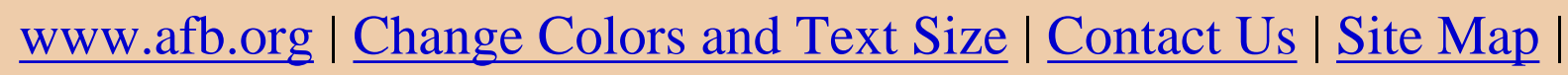
Site Search $\square$ Go

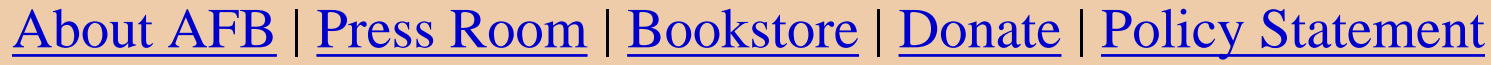

Please direct your comments and suggestions to afbinfo@afb.net Copyright (c) 2005 American Foundation for the Blind. All rights reserved. 